SHORT REPORT

\title{
The "pulvinar sign" in a case of paraneoplastic limbic encephalitis associated with non-Hodgkin's lymphoma
}

\author{
M Mihara, S Sugase, K Konaka, F Sugai, T Sato, Y Yamamoto, S Hirota, K Sakai, S Sakoda
}

J Neurol Neurosurg Psychiatry 2005;76:882-884. doi: 10.1136/jnnp.2004.049783

This paper reports a 59 year old woman with paraneoplastic limbic encephalitis associated with diffuse large B cell lymphoma. Her brain magnetic resonance imaging scan showed bilateral posterior thalamic hyperintensities, similar to the "pulvinar sign". Her symptoms included progressive psychiatric disturbance and resembled the initial symptoms of variant Creutzfeldt-Jakob disease (vCJD). Clinicians should consider this treatable disorder in the differential diagnosis of vCJD.

$\mathrm{P}$ araneoplastic neurological syndromes (PNS) are disorders of the nervous system that are associated with cancer but are not caused by the tumor growth itself or by non-metastatic complications such as secondary infections and metabolic, ischaemic or nutritional disorders. Paraneoplastic limbic encephalitis (PLE) is a subtype of PNS, characterised by personality changes, irritability, depression, seizures, memory loss and dementia. The most frequent magnetic resonance imaging (MRI) abnormalities in PLE are hyperintensity signals on T2-weighted or fluid attenuation inversion recovery (FLAIR) images involving one or both medial temporal lobes. ${ }^{12}$ Thalamic involvement has rarely been described. In this paper, we report the first case of PLE associated with non-Hodgkin's lymphoma showing bilateral hyperintensity signals in the posterior thalamus-known as the "pulvinar sign".

\section{CASE REPORT}

The patient was a 59 year old woman, who worked as a medical clerk. Her medical history was unremarkable except for a hysteromyoma. In October 2003, she noticed that she had lost $10 \mathrm{~kg}$ over a period of two months. She consulted a physician, and a gastroendoscopic examination revealed no abnormalities and routine laboratory examinations were unremarkable. However, her family observed that she had become more inactive and depressive than before. In February 2004, her colleagues noticed that she had become careless and inaccurate. She met with several minor car accidents, but she did not attach any significance to these. She became withdrawn and emotionally labile. In March 2004, she became forgetful and could not remember the password of her bank card for which she consulted a neurologist. Neurological examination revealed no motor or sensory disturbance; however, a brain MRI revealed high intensity signals in bilateral pulvinar on T2-weighted images (fig 1A). She was referred to the university hospital and admitted for further investigation.

At the time of admission, the physical examination was unremarkable and neurological examination revealed apathy, perseveration, attention deficit, and forgetfulness. The results of the neuropsychological examinations are shown in table 1. Involuntary movement, sensory disturbance, or cerebellar
Table 1 Neuropsychological examination results of the patient

\begin{tabular}{ll}
\hline Test battery (normal) & Result \\
\hline Mini Mental State Examination ( $>23$ ) & $24 / 30$ \\
Digit span (F $>5 \mathrm{~B}>3$ ) & Forward: F 6; backward: B 4 \\
Frontal Assessment Battery (>16) & $13 / 18$ \\
Wechsler Adult Intelligence Scale- & VIQ 83; PIQ 89; IQ 86 \\
Revised ( $>80$ ) & \\
Wisconsin Card Sorting Test & Achieved categories: CA 1 \\
$(\mathrm{CA}>4.2$ PE $<4.0)$ & Perseverative error: PE 11 \\
Verbal fluency & Non-perseverative error 5 \\
(letter $>7.6$ category $>13.6)$ & Letter 5/minute \\
\hline
\end{tabular}

ataxia were not evident. Routine blood and chemistry tests including $C$ reactive protein, vitamin $B_{1}$, and vitamin $B_{12}$ were normal. Serum virus screening test was negative for human immunodeficiency virus, human $\mathrm{T}$ lymphotrophic virus 1, herpes simplex and varicella zoster viruses, cytomegalovirus, Epstein-Barr virus, measles, hepatitis B and hepatitis C virus. Serum screening for syphilis was also negative. Thyroid functions were within the normal range. Antinuclear antibody was weakly positive $(\times 40)$, but other autoantibodies were negative. Immune electrophoresis was also normal. Neurone specific enolase was slightly elevated in the serum ( $13.7 \mathrm{ng} / \mathrm{ml})$, but other tumour markers including carcinoembryonic antigen, CA 19-9, CA 15-3, pro-gastrin releasing peptide and soluble interleukin-2 receptors were within the normal range. Cerebrospinal fluid (CSF) examination showed normal cell counts (4 lymphocytes/ $\mu$ l) without abnormal cells and elevated total protein $(56 \mathrm{mg} / \mathrm{dl})$ and IgG index (1.39). CSF oligoclonal bands were positive and 14-3-3 protein was negative. Serum and CSF screening for antineuronal antibodies were negative including anti-Hu, anti-Yo, anti-Ri, anti-Ta, anti-Ma, antiamphiphysin and CRMP-5. Electroencephalography revealed normal background activity with bilateral sporadic frontal spikes. Moreover, spike and wave activities, induced by photic stimulation, were also observed. Analysis of the prion protein gene revealed methionine homozygosity at codon 129 without any mutations.

Repeated brain MRI revealed high intensity signals in the left hippocampi in addition to bilateral posterior thalamus (fig 1B, C). A computed tomography (CT) scan of the chest revealed an enlarged left axillary lymph node. In addition, gallium citrate uptake was evident in the left axillary lymph node (fig 1D), which was dissected. Pathological examination revealed diffuse large B cell lymphoma (fig $1 E-G$ ). Adjuvant

Abbreviations: CSF, cerebrospinal fluid; MRI, magnetic resonance imaging; PLE, paraneoplastic limbic encephalitis; VCJD, variant Creutzfeldt-Jakob disease 

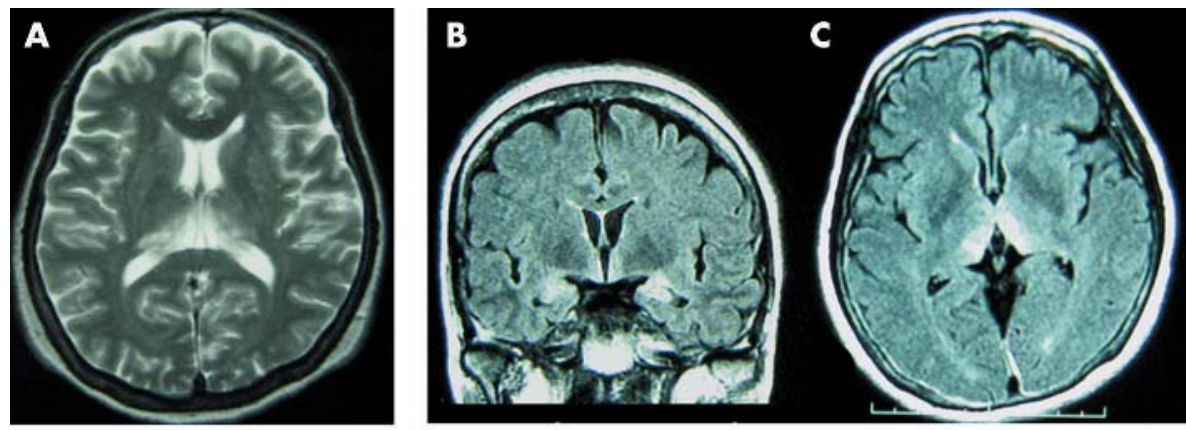

Figure 1 (A) The patient's first MRI obtained in March 2004. Axial T2weighted images $(T R / T E=3700 / 96)$ show bilateral posterior thalamic hyperintensities. (B, C) Follow up MRI images obtained after one month. Axial FLAIR image (B) (TI/TR/TE $=2000 /$ $8000 / 126$ ) showed bilateral medial and posterior thalamic hyperintensities. Coronal FLAIR images (C) revealed left dominant medial temporal lobe hyperintensities. (D) Gallium citrate scintigraphy revealed increased uptake at the left axillary lymph node. Liver and mucosal uptakes were normal, and
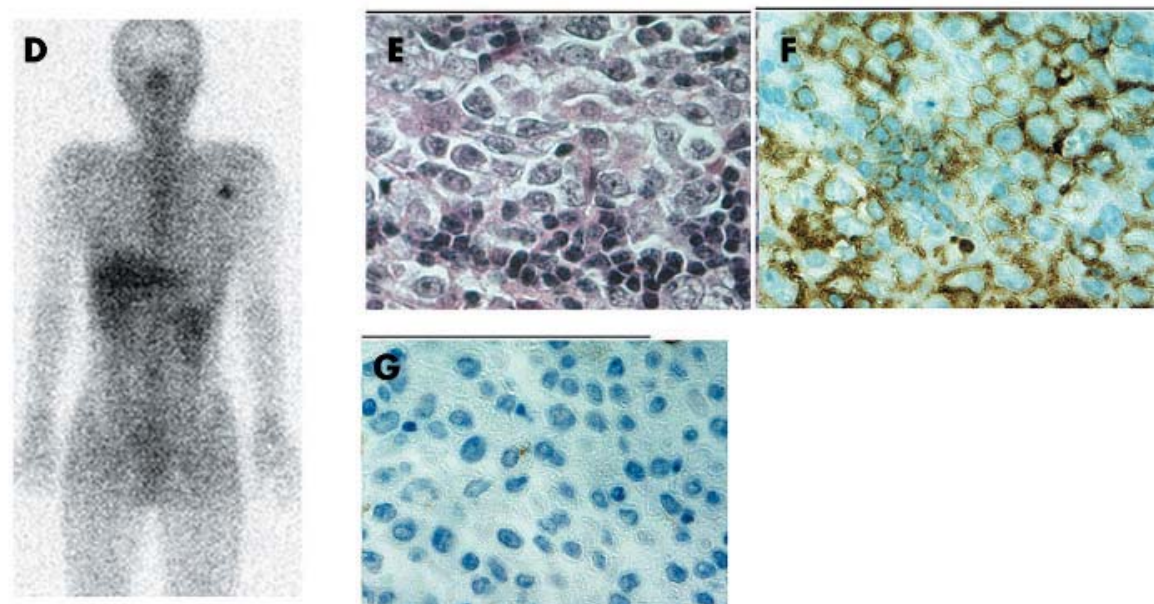
there was no evidence of cancer elsewhere in the body. (E-G) Biopsy of the left axillary lymph node exhibited proliferation of large lymphoid cells. Immunohistochemistry revealed that the cells were positive for LCA, CD20, and $C D 79 a$, but were negative for CD3 CD45R, CD43, S 100 protein, and cytokeratin. The findings were compatible with the diagnosis of diffuse large B cell lymphoma.

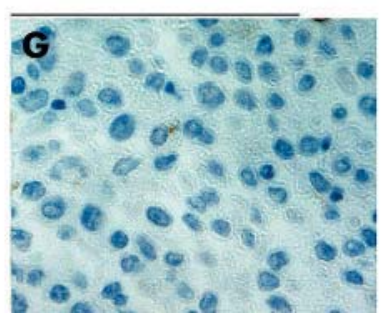

(E) Haematoxylin and eosin staining, $\times 400$. (F) Immunohistochemistry of CD20, $\times 400$. (G) Immunohistochemistry of CD3, $\times 400$.

chemotherapy was planned, but the patient refused to undergo chemotherapy. During the observation period of four months, she had no sign of recurrence of lymphoma and her neurological symptoms were stable.

\section{DISCUSSION}

PLE is a rare disorder and is hard to diagnose. The prominent clinical features of our patient were the neuropsychological symptoms that had developed over months. There was no evidence of intracranial malignancy, cerebrovascular disease, or metabolic abnormality. Only mild inflammatory changes were observed in the CSF without any abnormal cells. The lymphoma was localised to the left axillary lymph node. Further, the neurological symptoms did not deteriorate after the resection of the left axillary lymph node. These findings and the case history of the patient fulfil the diagnostic criteria of PLE, recently proposed by Gultekin and colleagues. ${ }^{2}$

Neoplasms commonly associated with PLE are carcinoma of the lungs, testis, and breasts. ${ }^{2}$ Association of nonHodgkin's lymphoma with PLE is rare, and only two cases have been reported previously. ${ }^{3}$ Furthermore, thalamic involvement, which is rare in PLE, was not observed in these cases. Scheid et al reported a case of PLE with breast cancer involving bilateral medial temporal lobes, cingulated gyrus, and thalamus. ${ }^{5}$ Nakahama et al reported a case of pathologically proved PLE predominantly involving the thalamus with gastric cancer. ${ }^{6}$ In a series of 50 cases studied by Gultekin and colleagues, ${ }^{2}$ only one patient exhibited thalamic involvement.

Bilateral symmetrical hyperintensity signals in the posterior thalamus are regarded as the "pulvinar sign", which is a characteristic of variant Creutzfeldt-Jakob disease (vCJD). ${ }^{7}$ The pulvinar sign in vCJD is defined as "bilateral, symmetrical pulvinar high signal relative to the signal intensity of other deep grey matter nuclei and cortical grey matter". ${ }^{8}$
Collie and colleagues reported that $85 \%$ of the initial MR images of patients with VCJD were positive for the pulvinar sign. They suggested that this sign became positive early in the disease course. They concluded that the sign is a sensitive and unique finding in vCJD. ${ }^{9}$ However, bilateral thalamic hyperintensity is not entirely specific to vCJD. ${ }^{7}$ Similar neuroradiological manifestations have been reported in several disorders including benign intracranial hypertension, ${ }^{10}$ Alpers' syndrome, ${ }^{11}$ acute encephalitis, ${ }^{12}$ post-infection encephalitis, ${ }^{13}$ Wernicke's encephalopathy ${ }^{14}$ and cat-scratch disease. ${ }^{15}$ However, clinical or other neuroradiological features have helped in distinguishing these disorders from vCJD.

Establishment of the surveillance case definition of vCJD could improve its clinical diagnostic accuracy, but early diagnosis of vCJD is still difficult because specific neurological features including sensory disturbance, cerebellar ataxia, and involuntary movement are often absent at disease onset and develop later. ${ }^{8}$ The present patient did not fulfil the case definition of VCJD at the time of admission, yet it was difficult to exclude the possibility of vCJD. The follow up MRI revealed hippocampal involvement, and the patient's symptoms ceased to deteriorate after the resection of the lymph node. These neuroradiological and clinical features are unlike those observed in VCJD and helped us suspect PLE. In addition, it should be noted that a patient with PLE could develop clinical features similar to those of vCJD including psychiatric symptoms, cerebellar ataxia, or sensory disturbances $^{2}$; a case of limbic encephalitis has been reported from among pathologically confirmed, misdiagnosed cases of vCJD. ${ }^{8}$ Therefore, a systematic examination including screening for malignancies and repeated cranial MRI should be considered for detecting this treatable differential diagnosis.

In summary, we report the first case of PLE with non-Hodgkin's lymphoma exhibiting the pulvinar sign on 
T2-weighted MRI. The exclusion of other possible disorders, especially treatable disorders such as PLE, is important in the differential diagnosis of vCJD. It is noteworthy that PLE may exhibit clinical and neuroradiological manifestations similar to those of vCJD.

\section{ACKNOWLEDGEMENTS}

We thank Professor K Doh-ura (Tohoku University) and Professor T Kitamoto (Tohoku University) for detection of 14-3-3 protein and analysis of the prion protein gene of the patient.

\section{Authors' affiliations}

M Mihara, S Sugase, K Konaka, F Sugai, T Sato, Y Yamamoto,

S Sakoda, Department of Neurology, Osaka University Graduate School of Medicine, Suita, Osaka, Japan

S Hirota, Department of Pathology, Osaka University Hospital, Suita, Osaka, Japan

K Sakai, Department of Neurology, Kanazawa Medical University, Ishikawa, Japan

Competing interests: none declared

Correspondence to: M Mihara, Department of Neurology, Osaka University Graduate School of Medicine,D-4, 2-2, Yamadaoka, Suita, Osaka 565-0871, Japan; m-mihara@neurol.med.osaka-u.ac.jp

Received 14 July 2004

Revised version received 11 September 2004

Accepted 15 September 2004

\section{REFERENCES}

1 Lawn ND, Westmoreland BF, Kiely MJ, et al. Clinical, magnetic resonance imaging, and electroencephalographic findings in paraneoplastic limbic encephalitis. Mayo Clin Proc 2003;78:1363-8.
2 Gultekin SH, Rosenfeld MR, Voltz R, et al. Paraneoplastic limbic encephalitis: neurological symptoms, immunological findings and tumour association in 50 patients. Brain 2000;123(Pt 7): 1481-94

3 Thuerl C, Muller K, Laubenberger J, et al. MR imaging of autopsy-proved paraneoplastic limbic encephalitis in non-Hodgkin lymphoma. AJNR Am J Neuroradiol 2003;24:507-11.

4 Javaheri S, Cohen V, Libman I, et al. Life-threatening tinnitus. Lancet 2000;356:308

5 Scheid R, Honnorat J, Delmont E, et al. A new anti-neuronal antibody in a case of paraneoplastic limbic encephalitis associated with breast cancer. J Neurol Neurosurg Psychiatry 2004;75:338-40.

6 Nakahama M. Fukusato T, Machinami R, et al. Paraneoplastic encephalomyelitis. An autopsy case with encephalitis principally confined to the thalamus and associated with pulmonary and gastric cancers. Acta Pathol Jpn 1990;40:371-5

7 Zeidler M SR, Collie DA, Knight R, et al. The pulvinar sign on magnetic resonance imaging in variant Creutzfeldt- Jakob disease. Lancet 2000;355:1412-18.

8 World Health Organization. The Revision of the Surveillance Case Definition for Variant Creutzfeldt-Jakob Disease (vCJD): Report of a WHO Consultation. Edinburgh: World Health Organization, 2001.

9 Collie DA, Summers DM, Sellar RJ, et al. Diagnosing variant CreutzfeldtJakob disease with the pulvinar sign: MR imaging findings in 86 neuropathologically confirmed cases. AJNR Am J Neuroradiol 2003;24:1560-9.

10 Yoshimura N, Soma Y. [A case of benign intracranial hypertension with bilateral reversible thalamic lesions on magnetic resonance imaging]. Rinsho Shinkeigaku 1992;32:327-9.

11 Harding BN, Alsanjari N, Smith SJ, et al. Progressive neuronal degeneration of childhood with liver disease (Alpers' disease) presenting in young adults. J Neurol Neurosurg Psychiatry 1995;58:320-5.

12 Kim SS, Chang KH, Kim KW, et al. Unusual acute encephalitis involving the thalamus: imaging features. Korean J Radiol 2001;2:68-74.

13 Cusmai R, Bertini E, Di Capua M, et al. Bilateral, reversible, selective thalamic involvement demonstrated by brain MR and acute severe neurological dysfunction with favorable outcome. Neuropediatrics 1994:25:44-7.

14 Weidauer S, Nichtweiss M, Lanfermann H, et al. Wernicke encephalopathy: MR findings and clinical presentation. Eur Radiol 2003;13:1001-9.

15 Hahn JS, Sum JM, Lee KP. Unusual MRI findings after status epilepticus due to cat-scratch disease. Pediatr Neurol 1994;10:255-8. 